\title{
HARMONIZATION OF RATIONAL AND CREATIVE DECISIONS IN QUALITY MANAGEMENT USING AI TECHNOLOGIES
}

\author{
Vytautas PALIUKAS ${ }^{1}$, Asta SAVANEVIČIENÉ ${ }^{2}$ \\ ${ }^{1}$ Panevezys College, Panevezys, Lithuania \\ ${ }^{2}$ Kaunas University of Technology, Kaunas, Lithuania \\ Corresponding author's e-mail:vytautas.paliukas@panko.lt
}

\begin{abstract}
Artificial Intelligence (AI) systems are rapidly evolving and becoming more common in management. Managers in business institutions are faced with the decision taking challenges and large amounts of data to be processed combining and harmonizing rational data with creative human experience in decision making.

The aim of the study is to reveal the main obstacles of the harmonization of creative and rational decisions making in quality management using AI technologies in the Quality Management System (QMS). The first section presents a literature review of approaches and trends related to AI technology usage in organisations for data processing and creative-rational decision making, rational and creative quality management decision making and paradigms in decision harmonization.

The Main Results section presents practical analysis and testing experience of automated AI Quality Management System developed at a higher education institution. During the analysis, an interview method was applied to find out specific system implementation issues. In the last section, the main analysis results and further development possibilities are discussed. The main findings and conclusions disclose two main problematic areas which may be defined as obstacles for rational and creative management decisions in quality management, related with clear responsibility distribution and assignment between data inputters and experience interpreters and duplicated qualitative data which $\mathrm{AI}$ system is not capable of rationalizing at the present development stage, speech and language processing techniques used when data processing algorithms cannot cope with the dual data processing technique, because in practice the system interprets and rationalizes only one category of data either quantitative based on rational defined indicators, or qualitative, based on language recognition and speech related data interpretation. Managers' experience in harmonizing creative human experience in organisation's quality management was evaluated as positive. Data processed by tested AI system allows for rationalization of creative experience with ready quantitative data output from QMS system and final harmonized strategic quality management decisions.
\end{abstract}

Keywords: Artificial Intelligence (AI), decision making, total quality management (TQM), quality management system (QMS).

JEL Classification: M10, M15 


\section{INTRODUCTION}

Strategic and tactical decisions by managers depend on abilities to harmonize rational and creative decision making. Using Artificial Intelligence (AI) technologies in contemporary organisations become more and more prevalent mainly due to the fact that these technologies prove to have abilities to reduce administrative work load and help to base decisions taken by managers on data instead of leaving them to take an intuitive decision making approach, but there paradigms arise related to rational and creative decision making. Simon (2013) states that it should be noted that administrative processes are decision processes: they consist in segregating certain elements in the decisions of members of the organisation, and establishing regular organisational procedures to select and determine these elements and to communicate them to the members concerned.

Decision making also includes communicative, creative and intuitive methods of decision communication and presentation. Rational decision making paradigm arises because a rational analysis is useful and necessary while making strategic decisions. But a rational approach is considered to be limited. One of the reasons is the fact that too little attention is paid to the influence of subjective factors on the process of decision making and its realization (Gudonavičius \& Savanevičiene, 2018).

Admitting that human being is not rational (Tversky \& Kahneman, 1974), we consider that rational and creative decision harmonization using AI technology may ensure needed rationality by providing the processed rational information for the managerial level, which then could be interpreted on a creative basis and human experience. AI systems and tools are used in various business management areas, one of which is quality management. The main advantages of AI usage for TQM in larger scale business organisations faced with large amounts of data needed to process every day are abilities to reduce the amount of human administrative work and provide managers with sufficient amount of comprehensive rational data needed for managerial decision - harmonizing human creative experience with rational data-based decisions. Most popular Quality Management Systems (QMSs) in the service organisations or institutions are usually based on general total quality management principles or international ISO certification requirements focusing mainly on control of specific service delivery processes, monitoring, auditing and gathering sufficient amount of the comprehensive rational data in order to make corrective managerial decisions based on rational data and managers' experience.

The study aims at revealing the main obstacles of the harmonization of creative and rational decisions making in quality management using AI technologies in QMS. The first section presents problematic approaches and trends of AI technology usage in organisations for data processing and creative-rational decision making. In the Main Results section, a practical analysis and testing experience of automated QMS system developed at a higher education institution are presented. In the last part, the main analysis results and further development possibilities are discussed. 


\section{METHODS AND PROCEDURES}

The study includes a critical literature analysis concerning AI system role in problem solving and rational-creative decision making paradigm and practical AI technology for quality management and analysis in a real system environment. In the scientific literature overview section, the usage of $\mathrm{AI}$ is discussed in management decision making, rational and creative decision making and rationalcreative decision harmonization AI techniques.

For the practical research, Computerized Quality Management System (CQMS) developed in 2015 by Panevėžys College (Lithuania) was chosen. This educational institution uses certified CQMS, which is regularly updated and certified according to ISO:9001 standard. Nature of the process-oriented service CQMS requires a lot of qualitative and quantitative data processing to make required quality management decisions. The College decided to implement the Management Information System (MIS) based on IT data processing and AI elements for quality management data analysis during the annual management cycle and strategic planning. AI intelligence tools in institutional MIS are used for quality management data accumulation and analysis. MIS undergoes the development stage.

Research was conducted using a qualitative research method. Structured qualitative interview was used to collect data from respondents in the educational institution using and developing QMS. During the analysis, an interview was conducted to find out specific system implementation issues for managers responsible for QMS data interpretation, input and quality management decision in the organisation decision making. Three main groups of obstacles for rational and creative decision harmonization were defined and presented in the interview questionnaires:

- distribution and assignment of responsibilities for data input;

- usage of language and speech related data processing techniques;

- harmonization of rational QMS system data output with creative managers' experience.

Interviewed managers were divided into two groups: those responsible for data management and input to QMS AI system and interpretation at the department level and top managers responsible for final strategic quality management decision making. Answers related to all three decision harmonization aspects were received from 5 department managers and 2 top managers; 7 persons participating in the TQM strategic decision making, involving AI QMS, were interviewed. Respondents' answers were grouped and analysed by making conclusions on the main groups of obstacles for rational and creative decision harmonization.

\subsection{AI in Decision Making / Literature Review}

Rapidly developing AI systems and their role in the management decision making are presenting a lot of new trends and possibilities for institution management. Information, AI and expert systems, which are designed to help managers in decision making, are becoming more and more popular in business and public institutions. Expert systems, designed to replicate certain abstract reasoning 
and problem-solving capabilities of humans (Simon \& Kaplan, 1989), are most appropriate in helping users to cope with semi-structured problems (Simon, 1977). Recent advances have made AI techniques accessible to a wider audience as seen by the increase in the number of applications in such areas as intelligent decision support systems. AI is used in decision support for tasks such as aiding the decision maker to select actions in real-time and stressful decision problems; reducing information overload, enabling up-to-date information, and providing a dynamic response with intelligent agents; enabling communication required for collaborative decisions; and dealing with uncertainty in decision problems (Phillips-Wren \& Jain, 2006). Decision making process is described by researchers who develop decision support systems as consisting of four phases: intelligence, design, choice and implementation. During the intelligence phase, the decision maker gathers information and develops an understanding of the problem. He/she identifies criteria, develops the model, and investigates alternatives during the design phase. A selection or decision is made during the choice phase, and the decision maker acts on the decision and learns during the implementation phase. The process proceeds in a generally sequential manner with feedback loops between phases.

Such systems usually consist of input, processing, and output to mirror the decision making process, but the decision maker is viewed as a crucial part of the overall system. These systems have the potential to be deeply embedded into the workspace and align more closely with the decision styles of users and the decision problem itself (Phillips-Wren, 2012). These systems are seen as "intelligent behaviour" indicators, which include:

- Learning or understanding from experience;

- Making sense out of ambiguous or contradictory messages;

- Responding quickly and successfully to a new situation;

- Using reasoning in solving problems;

- Dealing with perplexing situations;

- Understanding and inferring in ordinary and rational ways;

- Applying knowledge to manipulate the environment;

- Thinking and reasoning;

- Recognising the relative importance of different elements in a situation.

According to Tweedale, computer scientists aspire to amplify human reasoning within a virtual mind, ultimately emulating cognitive capabilities within silicon systems. Unfortunately, genuine know-how, wisdom and good judgement may be sacrificed in the process; however, explicit and logical automation will eventually emerge (Tweedale, 2014). Although the use of AI technologies continues to expand in the corporate world, the growth of the technology for the support of corporate or organisational strategic planning is somewhat slower (Spangler, 1991). To sum up, AI systems can help managers in logical decision making by processing and harmonising rational data with creative human experience.

\subsection{Rational and Creative Decision Making / Literature Review}

Lawler and Elliot (1996) in their experimental study of an expert system state that the knowledge base is highly complex and not readily accessible to those 
without specialized training. Consequently, organisations must rely on problem solvers who have accumulated a track record of generating solutions that, while not necessarily optimal, seem to work well. Expert problem solvers utilise heuristic, rather than algorithmic, methods. Dreyfus and Dreyfus (1986) indicates that human intuition is real and concludes that it usurps declarative or rule-based decisionmaking. They believe that "competent performance is rational; proficiency is transitional; but only experts act rationally". Rational data based decision making supposes an ethical and cultural impact. Oliveiro (2007) defines rationality as the compatibility between choice and value. Rational behaviour seeks to optimise the value of the outcomes focusing on the process of choosing rather than emphasising the selected alternative.

Jeff Catlin (2017), CEO of Lexalytics, suggests rather than putting AI in charge of ethical decision making, a better option is to train it to identify examples of ethical or unethical behaviour - and leave it up to humans to make decisions based on that categorization. Such an approach leads to understanding that people have both rational and creative decision-making models as their central element either as decision makers or as the ones who are affected by decisions. If such assumption is pertinent, then culture might play a role in the decision-making process (Oliveira, 2007).

In classical or perfect rationality, methods of decision analysis are used to attach numerical values or utilities to each of the alternatives during the "choice" phase. The alternative with the highest utility (or maximum subjective expected utility) is selected. When using the rational model, it is assumed that managers:

- know of all possible alternatives;

- know the consequences of implementing each alternative;

- have a well-organised set of preferences for these consequences;

- have the computational ability to compare consequences and to determine which is preferred (Kreitner \& Kinicki, 2001).

On the other hand, creativity in decision may lead to freedom mismanagement. Two types of executives mismanaging freedom in their decisions are discussed: first, managers tend to change goals frequently or fail to define them clearly. Employees may have freedom around process, but if they do not know where they are headed, such freedom is pointless. And, second, some managers fall short on this dimension by granting autonomy in name only. They claim that employees are "empowered" to explore the maze as they search for solutions but, in fact, "human is the creature of emotion" (Vaezipour, 2013). Consequently, in most of his organisational behaviour, including decision-making, plenty of feelings are involved. Therefore, in the process of aligning IT with business and further aligning business into the global economy considering the human factors would be essential for a successful and beneficial transmission. Rational approaches to decisionmaking can only be successful when human factors are well considered and carefully interacted within solution procedures. 


\subsection{AI Rational and Creative Decision Harmonization / Literature Review}

Rational is defined as "based on or in accordance with reason or logic", while choice is defined as "the act of choosing between two or more possibilities". Rational choice is a process of making decisions based on relevant information in a logical, timely and optimised manner. Today decisions are more and more made by artificially intelligent machines. These artificial intelligent machines bring several aspects that make the assumption of rationality stronger than when decisions are made by human beings. In this regard, the machine-made decisions are not as irrational as when they are made by human beings; however, they are not fully rational and therefore are still subjected to Herbert Simon's theory of bounded rationality (Marwala, 2017). Rational decision making steps commonly include perceiving the environment, invoking relevant information, then evaluating options and selecting an option with maximum utility.

The theory of rational choice states that given several options a rational human being will choose the option that maximises his utility (Becker, 1976; Hedström \& Stern, 2008; Grüne-Yanoff, 2012). This theory assumes several factors and these include the fact that the decision maker has all the information that he can use to generate several options. In addition, he can be able to construct all options as well as calculate the resulting utility for each option. The intuitive or creative decisionmaking model is also considered to be important for decision making. Opposite to rational decision making, this way refers to decision making without conscious reasoning. If strategic or tactical decisions are realised to be made with strict deadlines and time pressure, when it involves uncertainty and challenges it becomes clear that managers do not have enough time to go through all the rational decision making model steps.

All of the models include problem identification, which is the step in which the need for problem solving becomes apparent. Immersion is the step in which the decision maker thinks about the problem consciously and gathers information. During the incubation stage, an individual sets the problem aside. At this time, the brain is actually working on the problem unconsciously. Then comes illumination or the insight moment, when the solution to the problem becomes apparent to the person, usually when it is least expected. The verification and application stages happen when the decision maker consciously verifies the feasibility of the solution and implements the decision (Principles of Management, 2017).

Harmonizing rational data based decisions with human interpretation involves comprehensive data filtering: whenever information is irrelevant it needs to be marginalized or eliminated. Making decisions using information which contains irrelevant information often confuses a decision making process. We discuss four methods for making rational decisions by either marginalizing irrelevant information or not using irrelevant information. These methods are marginalization of irrationality approach, automatic relevance determination, principal component analysis and independent component analysis (Marwala, 2014). Harmonization of rational and creative decision making leads to the need of AI technique categorization and choice. Major categories of AI functionality defined are presented in Fig. 1. 


\begin{tabular}{|c|c|c|c|c|c|}
\hline \multicolumn{2}{|c|}{$\begin{array}{c}\text { Knowledge-Related } \\
\text { Techniques }\end{array}$} & \multicolumn{2}{|c|}{$\begin{array}{c}\text { Thought-Related } \\
\text { Techniques }\end{array}$} & \multicolumn{2}{|c|}{$\begin{array}{c}\text { Language-Related } \\
\text { Techniques }\end{array}$} \\
\hline 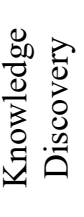 & 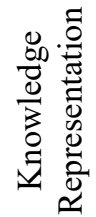 & 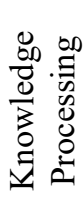 & 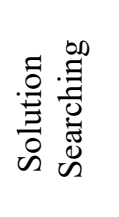 & 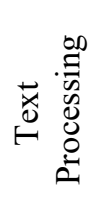 & 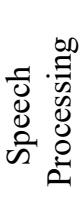 \\
\hline
\end{tabular}

Fig. 1. Categorization of major functionalities of AI (Strohmeier \& Piazza, 2015).

Knowledge discovery may also be reoffered to "machine learning", pattern recognition or "data mining, but in general it may be understood as the process of useful and valid information identification in data (Fayyad, Piatetsky-Shapiro, \& Smyth, 1996); knowledge representation means mapping of a set of relevant propositions to formal symbols in a way that allows computers to use these formal symbols when solving tasks (Brachman \& Levesque, 2004). For communication and decision making a very important stage in AI system is understanding language as the use of a complex system of spoken or encoded elements for communication; language usage constitutes a further clearly cognitive capability. Referring to language, text processing and speech processing constitute major categories of intelligent language-related techniques.

As it is possible to realise an AI application based on only one technique from one category, several techniques are increasingly combined and therefore constitute hybrid techniques (Kahraman, Kaya \& Çinar, 2010). Quality management in organisations is related to some specific feedback methods. To collect data and evaluate customer satisfaction, the ISO 10004 recommends using the following methods: personal interviews, phone interviews, discussion groups and mail surveys (Yussupova, Kovács, Boyko \& Bogdanova, 2016). However, these methods of collecting and analysing customer opinions show a number of significant drawbacks. A general drawback of the recommended methods is the need for a large amount of manual work: preparing questions, creating a respondent database, mailing questionnaires and collecting results, conducting personal interviews, preparing a report based on the results. All this increases the research costs.

It may be summed up that management decision making using AI for TQM must be harmonized between rational - data based and processed decision making and creative decision, using personal and sometimes even intuitive manager's experience for the final solution or strategic managerial decision. The set of several AI based techniques may be used; however, it is possible to realise AI applications using one of the mentioned techniques. 


\section{MAIN RESULTS}

AI system used in the analysed educational services providing institution with quality management clearly may be defined as a system mainly related to language based AI technique functionality because of the nature of data processing, which was implemented in the system. The main realisation principles, which were important for the organisation, were planned for the system implementation:

Usability. System was designed to be simple enough for users to use. During the realisation the main system's functions and modules were distributed using "tab" or "card" principles, including plans, analysis, processes, settings etc. AI tools used in the system output data for the managers in usable and understandable graphical format allow summarisation and creative decision harmonization with quantitative data. Accessibility. Access to the system is granted during 24 hours and 7 days per week. Confidentiality of the data is ensured by special security technological measures. Access to the system is granted only to the dedicated users with provided credentials - user names and passwords. System was designed assigning special rights (roles) to the users with a possibility to perform special actions (manage processes, organisational structure etc.). Data analysis is accessible for managers at anytime and anywhere. Scaling. The main system architecture and realisation support development and additional technical software and hardware. Protection from the main software and hardware interferences is implemented, such as power or internet connection disruptions.

System had to be complete and include all needed software for the user without the need of additional software installation. Technological platform ensures accessibility and functionality using popular and commonly used web browsers. Testing and analysis revealed that system was accessible by the users connected to the internet via Internet Explorer, Google Chrome, Mozilla Firefox and Opera. System was designed using standard data exchange interfaces and connections for information exchange with the possibilities to use "Web Services" mechanism for data import and export to external systems. System is able to use data export and import using most common data exchange formats such as "XML" and includes standard ETL (Extract, Transform, Load) tools for information indicator input, transformation and download to the main data base. Three-level architecture was implemented during system creation, including user's working place - the main service station - database management server.

Analysis of QMS data management revealed that system was planned to include subsystems for quality, staff and organization data management. The main planned subsystems in the computerized QMS system implemented in the College were as follows:

- Plans;

- Indicators;

- Organisational structure;

- Security management subsystem;

- Survey analysis and implementation;

- Process distribution and implementation;

- Information management; 
- Document management.

Analysis of the subsystems already implemented revealed that during the two years of subsystem implementation Planning, Indicators, Organisational Structure, Processes and Document Management subsystems were implemented at the comprehensive level, also including a survey analysis module. The system includes technological tools for external system integration, most important of which are finance management and study information accounting informational systems. Module principle was used in order to ensure additional functionality development possibilities. Testing of human error data inputting functionality revealed that wrong data input by the user was followed by comprehensive information action users were informed about data input mistakes by popping windows with the information on further actions needed and corrections.

The system also includes hierarchical data search on chosen criteria using data filtering ribbon with the possibilities to perform search on chosen indicators or plan elements. AI tools in the QMS are mostly implemented in the planning module. Planning subsystem module allows creating and registering in the system an unlimited number of plans such as strategical action, development etc. Every planning element can be assigned to a responsible person - manager, implementer etc. Indicator subsystem allows assigning and tracking special indicators and their definitions for every plan element and input planned indicator values, which can be tracked during the defined time periods. An important feature in the plan subsystem by the organisation is a possibility to create and present a chart for every indicator development in time and toleration intervals. Subsystem for organisational structure allows for registration of employees at every department by inputting complete information and assigning employees by the levels of their responsibilities and functions. This subsystem allows tracking organisation's hierarchical structure, data analysis and tracking.

Survey subsystem implemented in order to provide possibilities to gather data from organisation employees and to summarise data in real time allows presenting survey data in graphical forms and diagrams. Analysis of which system submodules are mostly used in the organisation has revealed that two subsystems are constantly in use - planning and document management. Most focus on the AI tools is made by the institution in the planning and document management submodules, which are further developed to allow more AI based interaction and learning in qualitative and quantitative data analysis. Planning subsystem was chosen for the AI technology in QMS usage analysis and interview, because all interviewed managers defined this subsystem as "most important" or "most needed" for the managerial quality management decision making in the organisation.

Top managers of the organisation explained that the main priority implementing the computerized QMS system was given to strategic planning implementation, devoting less attention to other modules along with the plans for future development. Most obstacles to retrieving and processing comprehensive decision-making data by the managers interviewed at the level of department managers responsible for data interpretation were mentioned in relation to responsibilities. Four of the interviewed managers being asked of the main problem related to data input to the system made clear comments on the responsibility issues, 
such as "I do not completely understand for which data indicators I am responsible" or, for example, "I am not directly responsible for the data I have to input and analyse".

Comments from the top managers on the responsibility also confirmed an obstacle to final decision harmonization in relation to responsibility, for example, "I notice several department managers inputting different data on the same indicator..." or "I see additional interpretative data from the manager who is not responsible for that particular area". The most credits were given to the document management subsystem (DMS), which allowed an organisation to make a smooth transition from paper document management to complete electronic document management, including tracking of electronic document preparation, administration stages and even signing a document by responsible persons electronically. Analysing the use of language and speech related data processing AI techniques, it should be mentioned that the whole system had to be implemented in the Lithuanian language following Lithuanian legal regulations concerning official documentation management in the national language. Some text and language processing, compatibility problems were noticed in relation to Lithuanian language encoding, locale, and font's specific.

The second group of obstacles preventing top managers from smooth rational and creative final quality management strategic decision taking was related to the functionality of language processing AI technique in the system. All five department managers interviewed in one or other context mentioned a problem concerning numerical and text related data processing in the system. Problem may be tracked analysing interview answers, such as "sometimes I found it hard to input or describe indicators in numerical values because of their qualitative nature", or "I rather prefer text descriptions than numerical indicators which are hard to calculate". Analysing AI QMS presented data, top managers noticed and commented as follows: "I cannot take a decision based on my creative experience if I get mixed, duplicating various format data from the AI system, like a lot of numeric quantitative indicators, which are mixed with qualitative text descriptions".

According to the persons responsible for planning subsystem data interpretation, the system presents the suggested plan implementation proposals for managers only if persons responsible for indicators, process or role implementation according to the hierarchical organisation system registered in the system submodule input the data are in the format, which is defined in the number or text processing fields. During the first year of data input and automated analysis it was noted that in many cases responsibilities for specific functions or indicators were duplicated allowing data administrators to enter several sometimes different numeric values or worse - free text interpretation in place of needed numeric values, which made the automated data analysis impossible.

As the system was designed in the way that quantitative indicators expressed in numeric calculated values could be added as qualitative text and language related subjective descriptions, the programmed AI algorithms are incapable of mixed text and numerical data processing, which results in a lot of unprocessed data that have to be recalculated and prepared for AI system processing manually This is also confirmed by the department managers expressing this problem as follows: "I have 
to manually recalculate indicators taking into consideration subjective or duplicating data comments before presenting data for top manager. This takes a lot time..." Such an incorrect and duplicated data input prevents the automated system from using AI algorithms for data processing, thus rendering comprehensive rational output for creative human interpretation and final quality management decision taking. The more discrepancies of such nature happen during the data input process, the more manual corrections are needed in the system in order to obtain rationally and correctly processed data. This may denote one of the main advantages of AI in QMS - the automated data processing and possibility to prepare rational data for harmonized decision making.

The third area of AI QMS based managerial decision making was expressed by the top managers' insights, which mainly included the main goals of the managers to reduce decision time and retrieve rational data. Top managers mentioned the main advantage of the system in the interview as follows: "I can track quality management data coming from the institution and follow rational indicators" and "I have enough quantitative data to make timely decisions harmonizing my experience and rational indicators". The positive attitude to the final decision making stage at the top management level was clearly expressed nevertheless making remarks that "system must be further developed shortening data processing and interpretation time".

\section{DISCUSSION AND CONCLUSIONS}

Theoretical analysis of rational and creative decision management and harmonization has revealed that AI systems and tools rapidly emerge as tools and techniques, which can be used to help decision makers - managers in the decision making and harmonization. Usage of AI in decision making and QMS systems reduces information overload, shortens decision making time and human workload on the information processing. On the other hand, taking into consideration that AI decision support or making system is a "human creation" itself, it becomes clear that a single approach only or rational or creative decision making is not possible due to present AI system limitations and data processing techniques. Conclusion is made that both rational and creative decision making models should be harmonized in order not to mismanage freedom, human emotional experience. For such harmonization, knowledge, thought and language related AI techniques are used.

Analysis of AI QMS implemented system for rational and creative decision harmonization, which was implemented using language related techniques, including elements of text processing and quantitative or qualitative indicator analysis using AI algorithms, leads to a conclusion revealing obstacles for decision harmonization, which include three main problematic areas. The first obstacle for comprehensive creative and rational decision making in organisation quality management leads to confirmation that AI systems and tools are created by the humans seeking not only rational experience, but also performing creative tasks. When AI system is implemented and used in a real organisation, it is expected to reduce time for data collection, interpretation and systematized data processing. Harmonization of rational and creative experience also leads to expectations for the 
AI to take part in the creative experience interpretation, which at the present stage of AI development is still not achievable. The main problem at the AI QMS "learning" stage or data input arises due to the need to define clearly human responsibilities for the data input. Analysis of managers' experience in an organisation implementing the QMS based on AI tools has revealed that a lack of clear responsibility distribution and assignment between data inputters and experience interpreters creates duplication of qualitative rather than quantitative data, which AI system is not capable of rationalizing. In such a case, the workload of administrative staff trying to prepare and interpret data increases. As an example, one of the "power quotes" of the department manager may be presented: "At the data input to QMS stage people responsible for this do not understand clearly which data they are responsible for. This creates duplicated entries and a lot of language related content entered as interpretation".

Nature of data processing AI techniques in the analysed AI QMS has revealed that the main AI functionality in this system may be defined as related to speech and language processing. AI data input techniques used in the analysed QMS seeking to collect quantitate data based on numerical indicators and qualitative data based on staff experience and creative interpretations create technical data processing and harmonization paradigm - AI tools including data processing algorithms cannot cope with the dual data processing technique because in practice the system interprets and rationalizes only one category of data either quantitativebased on rational defined indicators, or qualitative, based on language recognition and speech related data interpretation and rationalization. Manager responsible for data interpretation during the interview stated, "I have to interpret two kinds of different data - numbers and text because system is not able to do that". This paradigm can also be confirmed by a simple answer in the questionnaire stating that "the system does not recognise qualitative data and my written explanations. I have to input only numbers". As a result, in practice additional time consuming staff involvement for data coding and interpretation is needed. It may be discussed that the first two obstacles may be defeated by system architecture changes and AI tools learning capabilities development, but in case of the analysed organisation it may also be related to the lack of additional financing and research capabilities for existing and already created AI techniques implemented in the existing system.

The third field related to the top managers' experience in harmonizing their creative human experience in organisation's quality management may be evaluated as positive - if the first main obstacles are not taken into consideration or are corrected - top managers express usefulness of AI QMS system rational processed data for quality management in organisation decision making. The interviewed top manager of the organisation stated, "using this system I have sufficient quantitative data to make and justify my quality management decisions". Data processed by AI system allows for rationalization of creative experience with ready quantitative data output from QMS system and final harmonized strategic quality management decisions. 


\section{REFERENCES}

Becker, G.S. (1976). The Economic Approach to Human Behaviour. Chicago.

Brachman, R.J., Levesque, H.J. (2004). Knowledge Representation and Reasoning. Morgan Kaufmann, Burlington.

Catlin, J. (2017). The Role of Artificial Intelligence in Ethical Decision Making. Forbes. Retrieved from https:/www.forbes.com/sites/forbestechcouncil/2017/12/21/the-role-of-artificial-intelligence-in-ethicaldecision-making/\#5839833d21dc

Dreyfus, H. L., \& Dreyfus, S. D. (1986). Mind Over Machine: The Power of Human Intuition and Expertise in the Era of the Computer. Blackwell, Oxford, UK.

Fayyad, U., Piatetsky-Shapiro, G., \& Smyth, P. (1996). From Data Mining to Knowledge Discovery in Databases. AI Magazine, 17(3), 37-54.

Grüne-Yanoff, T. (2012). Paradoxes of Rational Choice Theory. In: Roeser S., Hillerbrand R., Sandin P., Peterson M. (eds) Handbook of Risk Theory. Springer, Dordrecht. https://doi.org/10.1007/978-94-0071433-5 19

Gudonavičius, L., \& Savanevičiene, A. (2018). Knowledge Organizations: Rational and Creative Thinking in Strategic Decision Making. Retrieved from https://www.researchgate.net/publication/228466245_Knowledge_Organizations_Rational_and_Creative Thinking_in_Strategic_Decision_Making

Hedström, P., \& Stern, C. (2008). Rational choice and sociology. In: Blume L. and Durlauf S. 2nd Edition. The New Palgrave Dictionary of Economics. 1-17. Retrieved from https://www.nuffield.ox.ac.uk/users/hedstrom/rct.pdf

Kahraman, C., Kaya, I., \& Çınar, D. (2010). Computational Intelligence: Past, Today and Future. In Ruan, D. (ed.), Computational Intelligence in Complex Decision Systems, Atlantis Press, Paris, 1-46.

Kreitner R \& Kinicki A. (2001). Organizational Behaviour. 5th Edition, Irwin McGraw Hill, Burr Ridge (IL).

Lawler J.J., \& Elliot, R. (1996). Artificial Intelligence in HRM: An Experimental Study of an Expert System. Journal of Management, 22(1), 85-111. https://doi.org/10.1177/014920639602200104

Marwala, T. (2014). Artificial Intelligence Techniques for Rational Decision Making. Springer. Advanced Information and Knowledge Processing. https://doi.org/10.1007/978-3-319-11424-8

Marwala, T. (2017). Rational Choice and Artificial Intelligence. Retrieved from https://arxiv.org/ftp/arxiv/papers/1703/1703.10098.pdf

Oliveira, A. (2007). Discussion of Rational and Psychological Decision-Making Theories and Models: The Search for a Cultural-Ethical Decision-Making Model. EJBO Electronic Journal of Business Ethics and Organization, 12(2). 12-17. Retrieved from http://ejbo.jyu.fi/pdf/ejbo_vol12_no2_pages_12-17.pdf

Phillips-Wren G., \& Jain, L. (2006). Artificial Intelligence for Decision Making. In: Gabrys B., Howlett R.J., Jain L.C. (eds) Knowledge-Based Intelligent Information and Engineering Systems. Lecture Notes in Computer Science, 4252, Springer, Berlin, Heidelberg, 531- 536. https://doi.org/10.1007/11893004 69

Phillips-Wren, Gloria, (2012). AI tools in decision making support systems: a review. International Journal on Artificial Intelligence Tools, 21(2), 1240005 (13 pages). World Scientific Publishing Company. https://doi.org/10.1142/S0218213012400052

Principles of Management. https://doi.org/10.24926/8668.1801

Simon, H.A. (2013). Administrative Behaviour. A study of decision-making processes in administrative organizations. 4th Edition. Simon and Schuster. The Free press. New York. 384.p.

Simon, H. A., \& Kaplan, C. A. (1989). Foundations of cognitive science. In M. I. Posner (Ed.), Foundations of cognitive science (pp. 1-47). Cambridge, MA, US: The MIT Press.

Simon, H.A. (1977). The New Science of Management Decision, (rev. ed.). Englewood Cliffs, NJ: PrenticeHall.

Spangler, W. (1991). The Role of Artificial Intelligence in Understanding the Strategic Decision-Making Process. IEEE Transactions on Knowledge and Data Engineering, 3(2), 149-159. https://doi.org/10.1109/69.87995

Strohmeier, S., Piazza, F. (2015). Artificial Intelligence Techniques in Human Resource Management-A Conceptual Exploration. In: Kahraman C., Çevik Onar S. (eds) Intelligent Techniques in Engineering Management. Intelligent Systems Reference Library, 87, Springer, Cham. 149-172. https://doi.org/10.1007/978-3-319-17906-3 7

Tversky, A., \& Kahneman, D. (1974). Judgment under Uncertainty: Heuristics and Biases. Science, New Series, 185(4157), 1124-1131. Retrieved from http://psiexp.ss.uci.edu/research/teaching/Tversky_Kahneman_1974.pdf

Tweedale, J.W. (2014). A Review of Cognitive Decision-making within Future Mission Systems, Procedia Computer Science, 35, 1043-1052, https://doi.org/10.1016/j.procs.2014.08.191

Vaezipour, A. (2013). Creativity in Decision-Making. Tech. Rep. 2013. Research Description, Jönköping University, School of Engineering. 
Yussupova N., Kovács G., Boyko M, \& Bogdanova D. (2016). Models and Methods for Quality Management Based on Artificial Intelligence Applications. Acta Polytechnica Hungarica, 13(3). 45-60. Retrieved from https://www.uni-obuda.hu/journal/Yussupova_Kovacs_Boyko_Bogdanova_67.pdf

\section{AUTHORS' SHORT BIOGRAPHIES}

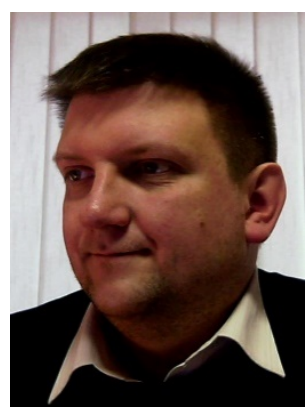

Vytautas PALIUKAS holds a Master degree in Business and Administration (2005). He is a Lecturer at Panevezys College, Lithuania, Head of Applied Research and Non-formal Education Department.

Research interests are related to artificial intelligence and management, TQM and strategic management, sustainable HRM.

E-mail: vytautas.paliukas@panko.lt

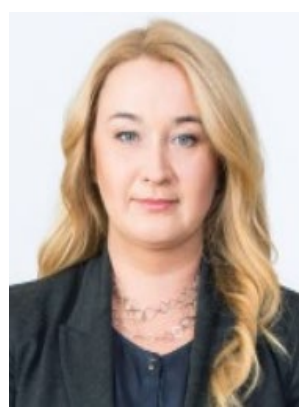

Asta SAVANEVIČIENE் holds Doctoral degree in Social Sciences (2000). She is a Professor Principal Investigator of Sustainable Management Research Group at the School of Economics and Business, Kaunas University of Technology, Lithuania. Research Interests include sustainable HRM and artificial intelligence.

E-mail: asta.savaneviciene@ktu.lt ORCID: https://orcid.org/0000-0003-3132-860X 